\section{Prevalência e fatores associados a fogachos em mulheres climatéricas e pós-climatéricas}

\author{
Prevalence and factors associated with hot \\ flashes in climacteric and post-climacteric women
}

\author{
1 Faculdade de Medicina, \\ Universidade Federal de \\ Pelotas, Pelotas, Brasil. \\ Correspondência \\ I. K. T. Sclowitz \\ Departamento de Medicina \\ Social, Faculdade de \\ Medicina, Universidade \\ Federal de Pelotas. \\ Av. Duque de Caxias 250, \\ Pelotas, RS, Brasil \\ ikt@conesul.com.br
}

\begin{abstract}
A cross-sectional study was conducted on the prevalence of hot flashes and associated factors among women from 40 to 69 years old, from a population-based sample in the city of Pelotas, southern Brazil. A multi-stage sampling process was used, and data were collected through an interview and a self-applied questionnaire. A total of 879 women were included. Point prevalence and lifetime prevalence were $30.1 \%$ and $53.2 \%$, respectively. Factors associated with hot flashes were: pre-menopausal status $(R P=2.33)$ and post-menopausal status $(R P=2.66)$; age 45 49 years $(R P=1.34)$ and $50-54$ years $(R P=1.42)$; lower social class $(R P=2.16)$; no use of a hormonal contraception method after 40 years of age $(R P=1.40)$; obesity $(R P=1.39)$ and overweight $(R P=1.32)$; and not having a partner $(R P=0.80)$. High prevalence of this symptom indicates that health facilities should be prepared to deal with problems encountered by climacteric women.
\end{abstract}

Climacteric; Hot Flashes; Menopause
Iândora Krolow Timm Sclowitz ${ }^{1}$

Iná da Silva dos Santos 1

Mariângela Freitas da Silveira 1

\section{Introdução}

De acordo com a Sociedade Internacional de Menopausa, o climatério é o período de transição entre a fase reprodutiva e a não reprodutiva da vida da mulher, podendo ser acompanhado de sintomas 1. Quando esses estão presentes, esse período pode ser designado "síndrome do climatério”. De acordo com a Organização Mundial da Saúde 2, o climatério compreende uma fase pré-menopausal, que começa em torno dos quarenta anos de idade e se estende até o início dos ciclos menstruais irregulares e/ou de sintomatologia atribuível à falência ovariana, com duração variável; uma fase peri-menopausal, que se inicia anteriormente à menopausa, com o começo dos sintomas, e se estende até o primeiro ano após a menopausa; e uma fase pós-menopausal, que se inicia após a parada da menstruação e se estende até os 65 anos de idade, englobando a fase pósmenopáusica da peri-menopausa. Após o climatério, tem início a fase pós-climatérica. Cronologicamente, o evento demarcador das fases do climatério é a menopausa, definida como a ausência da menstruação por, pelo menos, 12 meses consecutivos. Os limites de idade para climatério e menopausa ainda são bastante controversos 2 .

À síndrome do climatério tem sido atribuída uma ampla gama de sintomas. Os sintomas de origem neurogênica são os mais comuns e 
incluem a queixa mais prevalente nessas mulheres, que são os fogachos. Estudos mostram prevalências de fogachos variando de 18,0\% 3 a $74,0 \% 4$, de acordo com a população estudada.

Os fogachos consistem em sensação súbita e transitória de calor moderado ou intenso, que se espalha pelo tórax, pescoço e face, podendo ser acompanhados de sudorese profusa e sendo piores à noite. Durante a onda de calor, há elevação da temperatura corporal.

Os fogachos acometem mulheres em climatério ou em pós-climatério, podendo causar desconforto e ser motivo de muitas consultas médicas 2. No Brasil, a população feminina, de 40 a 69 anos, em 1993, era de cerca de 15,7 milhões. No ano de 1996, o contingente de mulheres, nessa faixa etária, passou para mais de 18 milhões (Departamento de Informática do SUS: http://www.datasus.gov.br, acessado em 15/Jul/2001). Com o aumento da expectativa de vida, há um número crescente de mulheres vivendo em climatério e menopausa, expostas, portanto, potencialmente, ao sintoma. Os fatores de risco conhecidos para a ocorrência de fogachos incluem: idade da menopausa 5 , baixa escolaridade 5,6; idade 6,7, estado menopausal $4,5,8,9,10$, cor da pele 11 , trabalho remunerado 10 , tabagismo 9,10,12 e índice de massa corporal (IMC) 4,9,13. O conhecimento pelos médicos e pacientes dos fatores de risco para os fogachos pode auxiliar na prevenção e no manejo adequado da síndrome do climatério. O presente estudo teve como objetivo avaliar a prevalência de fogachos e os fatores associados com a sua ocorrência, entre mulheres de 40 a 69 anos de idade, residentes na área urbana de Pelotas, Rio Grande do Sul, Brasil.

\section{Metodologia}

Este estudo faz parte de um consórcio de pesquisa, com participação de 11 mestrandos em Epidemiologia da Universidade Federal de Pelotas, com objetivos e projetos diferentes, unidos para a realização da coleta de dados, pois todos os trabalhos foram de base populacional, com dados sócio-econômicos comuns. Com o consórcio, usou-se somente um questionário com as variáveis de interesse para cada mestrando. Resultados desse consórcio podem ser encontrados em outras publicações 14,15,16,17,18. Foi um estudo transversal, incluindo, na amostra, a seleção de mulheres de 40 a 69 anos.

Pelotas é um município localizado ao sul do Rio Grande do Sul, com população, em 2001, de 323.158 habitantes, dos quais 169.816 são mulheres. $\mathrm{O}$ tamanho da amostra foi calculado para alfa de $5,0 \%$, poder de $80,0 \%$, prevalência esperada de fogachos de $18,0 \%$, a mais baixa encontrada, de acordo com o estudo de Tang 3 , com margem de erro de três pontos percentuais, totalizando 630 mulheres. Acrescido de $10,0 \%$ para perdas e recusas, seria necessário estudar 693 mulheres. Sendo de 0,6 o número de mulheres de 40 a 69 anos de idade, por domicílio, seria necessário visitar 1.155 domicílios. Para atender a necessidade do consórcio, foram selecionados 1.600 domicílios.

O processo de amostragem foi realizado em múltiplos estágios, a partir dos 281 setores censitários utilizados pelo Instituto Brasileiro de Geografia e Estatística 19. Sortearam-se 80 setores, em quatro diferentes estratos de escolaridade do chefe de família: (a) instrução inferior a quatro anos; (b) quatro a menos de oito anos; (c) oito a menos de 11 anos; e (d) 11 anos ou mais. Foram visitados vinte domicílios por setor. Apesar da amostragem complexa, as medidas de ocorrência e de efeito não necessitaram correção para o efeito de delineamento, uma vez que esse foi da ordem de 0,86. O trabalho de campo foi realizado de 25 de fevereiro de 2002 a 10 de maio de 2002.

Os dados foram coletados através de entrevista e de um instrumento auto-aplicável, confidencial, numerado, preenchido pela entrevistada e colocado em envelope pardo, lacrado, o qual era aberto e codificado somente pela pesquisadora responsável. Quando as mulheres não eram alfabetizadas, a própria entrevistadora aplicava o questionário confidencial.

As variáveis coletadas foram idade (em anos completos, de acordo com a data de nascimento), cor da pele (observada pela entrevistadora), situação conjugal (com ou sem companheiro), escolaridade (em anos completos de estudo), trabalho remunerado da mulher ( $\operatorname{sim} /$ não), renda própria da mulher (coletada em reais e, posteriormente, transformada em salários mínimos, de acordo com o piso nacional) e status sócio-econômico (pela classificação da Associação Nacional de Empresas de Pesquisa 20). Calculou-se o IMC através do peso e altura referidos pela entrevistada.

As variáveis reprodutivas estudadas foram idade da menarca (anos completos) e a data da última menstruação, que foi base para a determinação do status menopausal. A amostra foi dividida em três grupos de acordo com o status menopausal: as mulheres acima de 65 anos foram classificadas em pós-climatério, e as restantes foram divididas em pós-menopáusicas, se haviam parado de menstruar há mais de um ano, ou em pré-menopausa nas demais situações. 
As variáveis comportamentais investigadas foram início da atividade sexual (coletada através do questionário auto-aplicado), uso de método anticoncepcional hormonal após os quarenta anos de idade (sim/não), hábito de fumar (sim/não/ex-fumante) e uso de tratamento para menopausa (sim/não/já usou).

O desfecho foi investigado pela variável fogacho ( $\mathrm{sim} /$ não/já sentiu). No instrumento de coleta de dados, foi usado o termo "calorões", em lugar de fogachos. A equivalência semântica do termo "calorões" foi avaliada em uma amostra de 122 mulheres, antes do início de trabalho de campo. Mulheres com idade acima de quarenta anos, que aguardavam consulta na sala de espera em três Postos de Saúde da cidade, foram questionadas sobre a provável idade da menopausa e sintomas que podem ocorrer nesse período. Apenas 1,6\% das mulheres não tinha conhecimento da idade provável de parada da menstruação, as demais referiram uma média de 43,2 anos. Quarenta diferentes sintomas foram relatados, sendo que $75,4 \%$ das mulheres citaram os "calorões" como sintoma de menopausa. Outros sintomas referidos foram cefaléia $(17,2 \%)$ e nervosismo $(9,8 \%)$.

A prevalência ponto de fogachos foi definida como a proporção de mulheres atualmente com o sintoma. A prevalência em algum momento da vida (prevalência de episódio) foi definida como a proporção de mulheres que responderam "sim" ou "já sentiu" fogachos. A freqüência do desfecho entre as mulheres atualmente sintomáticas foi definida através do número de dias na semana com ocorrência de fogachos e da presença do sintoma na última semana. O número médio de episódios de fogachos, na semana anterior à entrevista, foi utilizado para a criação de um escore de freqüência do sintoma: leve (1-3 episódios), moderada (4-9) ou intensa (10 ou mais).

Para o controle de qualidade, foram reentrevistadas $13,1 \%$ da amostra, no menor tempo possível, não excedendo sete dias. As análises bivariada e multivariada foram realizadas no programa Stata 7.0. As razões de prevalências (RP) foram obtidas por regressão de Poisson com variância robusta, uma vez que o desfecho era binário e freqüente 21 . Na análise multivariada, as variáveis foram introduzidas conforme modelo de análise definido a priori (Figura 1). Para cada nível, realizou-se regressão por eliminação retrógrada, descartando variáveis com $\mathrm{p}>0,20$. O $\mathrm{p} \leq 0,20$ foi escolhido para incluir as variáveis de confusão que poderiam influir nas estimativas.

O estudo foi aprovado pela Comissão de Ética em Pesquisa da Faculdade de Medicina da Universidade Federal de Pelotas, tendo sido garantido sigilo das informações e obtido consentimento verbal das mulheres entrevistadas, antes da coleta de dados.

\section{Resultados}

Nos 1.600 domicílios visitados, foram identificadas 917 mulheres na faixa etária de 40 a 69 anos. Apenas 38 mulheres recusaram-se a dar entrevista ou não puderam ser localizadas após o mínimo de três tentativas. Dessas 38, não se dispõe de informação sobre a idade de quatro mulheres. Para o controle de qualidade, foram revisitadas 115 mulheres, para as quais foi repetida a questão sobre idade da menarca, obtendo-se kappa de 0,78.

A Tabela 1 mostra as características sócioeconômicas e demográficas da amostra e a prevalência ponto de fogachos, de acordo com essas características. A média de idade das mulheres entrevistadas foi de 52,1 $\pm 8,2$ anos. Mais de $80,0 \%$ eram de cor branca, e $61,3 \%$ viviam com companheiro. Quanto à escolaridade, a média foi de $6,6 \pm 4,5$ anos, havendo $9,7 \%$ de analfabetas e 14,1\% com 12-18 anos de educação formal. Em torno de $45,0 \%$ das mulheres desempenhavam alguma atividade remunerada, com mediana salarial de $\mathrm{R} \$ 293,00$ mensais, sendo que $11,4 \%$ ganhavam meio salário mínimo ou menos por mês. Quanto ao status sócio-econômico, cerca de $40,0 \%$ estavam nas classes D e E.

Quanto à prevalência ponto de fogachos, a faixa etária que mais relatou essa sintomatologia foi a das mulheres de 50 a 54 anos de idade (41,2\%; IC95\%: 34,1-48,4). Em termos de cor da pele, a maior prevalência foi entre as não brancas $(39,6 \%)$. As mulheres que viviam com companheiro relatavam mais fogachos $(32,4 \%)$ do que as demais (26,3\%). Quanto à escolaridade, os fogachos foram mais freqüentes à medida que diminuía o número de anos de educação formal da mulher, sendo de $19,5 \%$ entre aquelas com 12 a 18 anos de escola e de $35,6 \%$ entre as com 1 a 4 anos. A prevalência ponto de fogachos foi muito semelhante entre mulheres que trabalhavam $(29,5 \%)$ e as que não tinham atividade remunerada (30,5\%). Em termos de renda própria da mulher, a maior prevalência foi observada entre as que recebiam 0,6-1 salários mínimos mensais $(38,4 \%)$, e a menor $(21,8 \%)$, entre as que recebiam mais de cinco. As mulheres do status sócio-econômico E relatavam a presença atual de fogachos cerca de duas vezes mais freqüentemente $(35,0 \%)$ do que as da classe A $(18,2 \%)$. 
Figura 1

Modelo de análise.

1ㅇ nível

Status menopausal

2o nível $\quad$\begin{tabular}{c|c|} 
Demográficas \\
Idade \\
Cor da pele \\
Situação conjugal
\end{tabular}$\quad \begin{gathered}\text { Sócio-econômicas } \\
\text { Trabalho remunerado } \\
\text { Renda da mulher } \\
\text { Status sócio-econômico (ANEP) } \\
\text { Escolaridade }\end{gathered}$

\begin{tabular}{|c|c|c|}
\hline 3o nível & $\begin{array}{c}\text { Biológica } \\
\text { Índice de massa corporal }\end{array}$ & $\begin{array}{c}\text { Comportamentais } \\
\text { Início da atividade sexual } \\
\text { Uso de anticoncepção hormonal } \\
\text { Hábito de fumar }\end{array}$ \\
\hline
\end{tabular}

4o nível \begin{tabular}{c|} 
Reprodutiva \\
Idade da menarca
\end{tabular}

5ㅇ nível

Fogachos

ANEP = Associação Nacional de Empresas de Pesquisa.

A Tabela 2 descreve características biológicas, comportamentais e de manejo médico. Para 475 mulheres $(54,5 \%)$, a última menstruação havia ocorrido há mais de um ano. A média de idade da menopausa foi de 46,2 $\pm 6,0$ anos. Quanto ao status menopausal, 46,1\% estavam em pós-menopausa (última menstruação há mais de um ano e idade menor que 65 anos); e 397 (45,5\%) ainda menstruavam ou haviam parado há menos de um ano, sendo classificadas como em pré-menopausa. Cerca de $8,0 \%$ das entrevistadas tinham mais de 65 anos de idade e foram classificadas como pós-climatéricas. A prevalência ponto de fogachos associou-se fortemente com o status menopausal $(\mathrm{p}=0,006)$, sendo maior $(33,3 \%)$ entre as mulheres em pósmenopausa. Entre as pré-menopáusicas, a prevalência ponto foi de $29,2 \%$. Cerca de $12,5 \%$ das mulheres em pós-climatério relataram a presença do sintoma.

Mais da metade das mulheres $(51,2 \%)$ tiveram a primeira menstruação entre 13 e 15 anos (média de 12,8 \pm 1,8 ano) e iniciaram a atividade sexual entre 16 e 20 anos (média de 20,2 \pm 4.6 anos). Foi relatado o uso de anticoncepção hormonal, após os quarenta anos de idade, por $27,4 \%$ das entrevistadas. Quanto ao hábito de fumar, mais da metade nunca fumou. Eram fumantes e ex-fumantes, respectivamente, $26,0 \%$ e $18,9 \%$ das entrevistadas. De acordo com o IMC, cerca de $45,0 \%$ das mulheres apresentavam peso normal, sendo as demais classificadas como com sobrepeso $(34,4 \%)$ ou obesidade $(21,1 \%)$. Dessas características, somente o hábito de fumar e o índice de massa corporal apresentaram associação estatisticamente significativa com a presença do sintoma. As fumantes relataram fogachos mais freqüentemente $(36,7 \%)$ do que as ex-fumantes $(31,9 \%)$ e as que nunca fumaram $(26,4 \%)$. As mulheres com IMC normal (abaixo de 25) relataram menos freqüentemente a presença do sintoma $(24,8 \%)$ do que as com sobrepeso $(32,9 \%)$ e as obesas $(34,7 \%)$. 
Descrição da amostra de mulheres de 40-69 anos de idade e prevalência pontual de fogachos, de acordo com características sócio-econômicas e demográficas. Pelotas, Rio de Janeiro, Brasil, 2002.

\begin{tabular}{|c|c|c|c|}
\hline Variável & $\mathbf{n}$ & $\%$ & $\begin{array}{l}\text { Prevalência do } \\
\text { desfecho (IC95\%) }\end{array}$ \\
\hline \multicolumn{4}{|c|}{ Idade (anos completos) $(\mathrm{n}=879)$} \\
\hline \multicolumn{4}{|c|}{ Média \pm DP $=52,1 \pm 8,2$} \\
\hline $40-44$ & 192 & 21,8 & $25,7 \%(19,5-32,3)$ \\
\hline $45-49$ & 184 & 20,9 & $34,6 \%(27,4-41,6)$ \\
\hline $50-54$ & 192 & 21,8 & $41,2 \%(34,1-48,4)$ \\
\hline $55-59$ & 128 & 14,6 & $29,1 \%(21,2-36,6)$ \\
\hline $60-64$ & 92 & 10,5 & $23,3 \%(14,7-32,7)$ \\
\hline $65-69$ & 91 & 10,4 & $14,4 \%(7,8-23,2)$ \\
\hline \multicolumn{4}{|l|}{ Cor da pele $(n=879)$} \\
\hline Branca & 733 & 83,4 & $28,2 \%(24,7-31,4)$ \\
\hline Não branca & 146 & 16,6 & $39,6 \%(31,1-47,4)$ \\
\hline \multicolumn{4}{|c|}{ Situação conjugal ( $\mathrm{n}=879$ ) } \\
\hline Com companheiro & 539 & 61,3 & $32,4 \%(28,2-36,2)$ \\
\hline Sem companheiro & 340 & 39,7 & $26,3 \%(21,6-31,2)$ \\
\hline \multicolumn{4}{|c|}{ Escolaridade (anos) $(n=877)^{\star}$} \\
\hline \multicolumn{4}{|c|}{ Média $\pm \mathrm{DP}=6,6 \pm 4,5$} \\
\hline $12-18$ & 124 & 14,1 & $19,5 \%(12,8-27,4)$ \\
\hline $9-11$ & 136 & 15,5 & $28,9 \%(21,2-37,0)$ \\
\hline $5-8$ & 297 & 33,9 & $32,5 \%(27,0-38,0)$ \\
\hline $1-4$ & 235 & 26,8 & $35,6 \%(29,2-41,8)$ \\
\hline 0 & 85 & 9,7 & $23,8 \%(15,0-34,0)$ \\
\hline \multicolumn{4}{|c|}{ Trabalho remunerado $(n=879)$} \\
\hline Sim & 400 & 45,5 & $29,5 \%(24,8-34,0)$ \\
\hline Não & 479 & 54,5 & $30,5 \%(26,2-34,6)$ \\
\hline \multicolumn{4}{|c|}{ Renda da mulher (salários mínimos) $(\mathrm{n}=387)^{\star \star}$} \\
\hline$\geq 5,1$ & 56 & 14,5 & $21,8 \%(11,6-34,4)$ \\
\hline $3,1-5$ & 44 & 11,4 & $27,3 \%(14,9-42,8)$ \\
\hline $1,1-3$ & 143 & 36,9 & $25,9 \%(18,9-33,8)$ \\
\hline $0,6-1$ & 100 & 25,8 & $38,4 \%(28,5-48,2)$ \\
\hline$\leq 0,5$ & 44 & 11,4 & $34,9 \%(20,5-49,9)$ \\
\hline \multicolumn{4}{|c|}{ Status sócio-econômico (ANEP) $(n=877)^{\star}$} \\
\hline A & 44 & 5,0 & $18,2 \%(8,2-32,7)$ \\
\hline B & 158 & 18,0 & $20,4 \%(14,3-27,4)$ \\
\hline C & 332 & 37,9 & $31,6 \%(26,4-36,6)$ \\
\hline D & 303 & 34,5 & $34,7 \%(29,0-40,0)$ \\
\hline E & 40 & 4,6 & $35,0 \%(20,6-51,7)$ \\
\hline
\end{tabular}

IC95\% = intervalo de confiança de 95\%; DP = desvio-padrão; ANEP = Associação Nacional de Empresas de Pesquisa. * Houve perda de informação da variável.

** Apenas mulheres com trabalho remunerado. 
Descrição da amostra de mulheres de 40-69 anos de idade e prevalência pontual de fogachos, de acordo com variáveis biológicas, comportamentais e de manejo médico. Pelotas, Rio Grande do Sul, Brasil, 2002.

\begin{tabular}{|c|c|c|c|}
\hline Variável & n & $\%$ & $\begin{array}{c}\text { Prevalência de } \\
\text { fogachos (IC95\%) }\end{array}$ \\
\hline \multicolumn{4}{|c|}{ Status menopausal $(\mathrm{n}=872)^{\star}$} \\
\hline Pós-climatério & 73 & 8,4 & $12,5 \%(5,8-22,1)$ \\
\hline Pós-menopausa & 402 & 46,1 & $33,3 \%(28,7-38,2)$ \\
\hline Pré-menopausa & 397 & 45,5 & $29,2 \%(24,5-33,7)$ \\
\hline \multicolumn{4}{|c|}{ Início da atividade sexual (anos) $(n=821)^{\star}$} \\
\hline \multicolumn{4}{|c|}{ Média $\pm D P=20,2 \pm 4,6$} \\
\hline$<15$ & 75 & 9,2 & $36,0 \%(25,2-47,9)$ \\
\hline $16-20$ & 446 & 54,3 & $30,7 \%(26,2-35,0)$ \\
\hline $21-25$ & 211 & 25,7 & $27,0 \%(21,1-33,5)$ \\
\hline$>26$ & 89 & 10,8 & $28,4 \%(19,1-38,6)$ \\
\hline \multicolumn{4}{|c|}{ Uso de anticoncepção hormonal (após 40 anos) $(n=872)^{\star}$} \\
\hline Sim & 239 & 27,4 & $25,2 \%(19,7-31,1)$ \\
\hline Não & 633 & 72,6 & $31,9 \%(28,1-35,5)$ \\
\hline \multicolumn{4}{|c|}{ Hábtio de fumar $(n=879)$} \\
\hline Não & 484 & 55,1 & $26,3 \%(22,2-30,2)$ \\
\hline Sim & 229 & 26,0 & $36,7 \%(30,0-42,8)$ \\
\hline Ex-fumante & 166 & 18,9 & $31,9 \%(24,9-39,6)$ \\
\hline \multicolumn{4}{|c|}{ Índice de massa corporal (referido atual) $(n=823)^{\star}$} \\
\hline Normal $(<25)$ & 366 & 44,5 & $24,8 \%(20,3-29,3)$ \\
\hline Sobrepeso (25-29) & 283 & 34,4 & $32,9 \%(27,1-38,3)$ \\
\hline Obeso $(>30)$ & 174 & 21,1 & $34,7 \%(27,4-42,0)$ \\
\hline \multicolumn{4}{|c|}{ Uso de tratamento para menopausa $(n=856)^{\star}$} \\
\hline Não & 615 & 71,8 & $27,2 \%(23,7-30,8)$ \\
\hline Sim & 114 & 13,1 & $38,6 \%(29,6-48,2)$ \\
\hline Uso passado & 127 & 15,1 & $39,4 \%(30,8-48,4)$ \\
\hline \multicolumn{4}{|c|}{ Idade da menarca (anos) $(n=862)^{\star}$} \\
\hline \multicolumn{4}{|c|}{ Média $\pm \mathrm{DP}=12,8 \pm 1,8$} \\
\hline $16-18$ & 52 & 6,0 & $21,2 \%(11,1-34,7)$ \\
\hline $13-15$ & 441 & 51,2 & $30,3 \%(25,9-34,7)$ \\
\hline $8-12$ & 369 & 42,8 & $31,6 \%(26,7-36,4)$ \\
\hline
\end{tabular}

IC95\% = intervalo de confiança de 95\%; DP = desvio-padrão.

* Variável com perda de informações.

Quanto ao tratamento para menopausa, $72,0 \%$ das mulheres relataram que nunca fizeram uso. Entre as que usaram ou ainda usavam, a idade média de início do tratamento foi de 46,5 $\pm 6,1$ anos. O tempo médio de tratamento entre as ex-usuárias foi de 2,8 $\pm 4,7$ anos.

A Figura 2 descreve a prevalência ponto do sintoma fogacho. Cerca de $30,0 \%$ das mulheres referiram a presença atual do sintoma. Das 610 mulheres que não apresentavam, atualmente, o sintoma, 202 referiram já haver sentido, no passado, fogachos. Dessa forma, a prevalência em algum período da vida (prevalência de episódio), incluindo as mulheres atualmente sintomáticas e as que tiveram o sintoma no passado, foi de $53,2 \%$. A média de idade do início dos fogachos foi de 44,6 $\pm 5,7$ anos; e o tempo médio de duração do sintoma, entre as mulheres sintomáticas no passado, foi de 4,5 \pm 5,5 anos. Dentre as mulheres atualmente sintomáticas, 50,0\% apresentavam fogachos diariamente. Na última semana, $77,4 \%$ das mulheres apresentaram fogachos, sendo que $78,3 \%$ dessas referiram um a três episódios por dia (forma leve). 
Prevalência pontual e freqüência de fogachos atuais em mulheres de 40-69 anos de idade.

Pelotas, Rio Grande do Sul, Brasil, 2002.

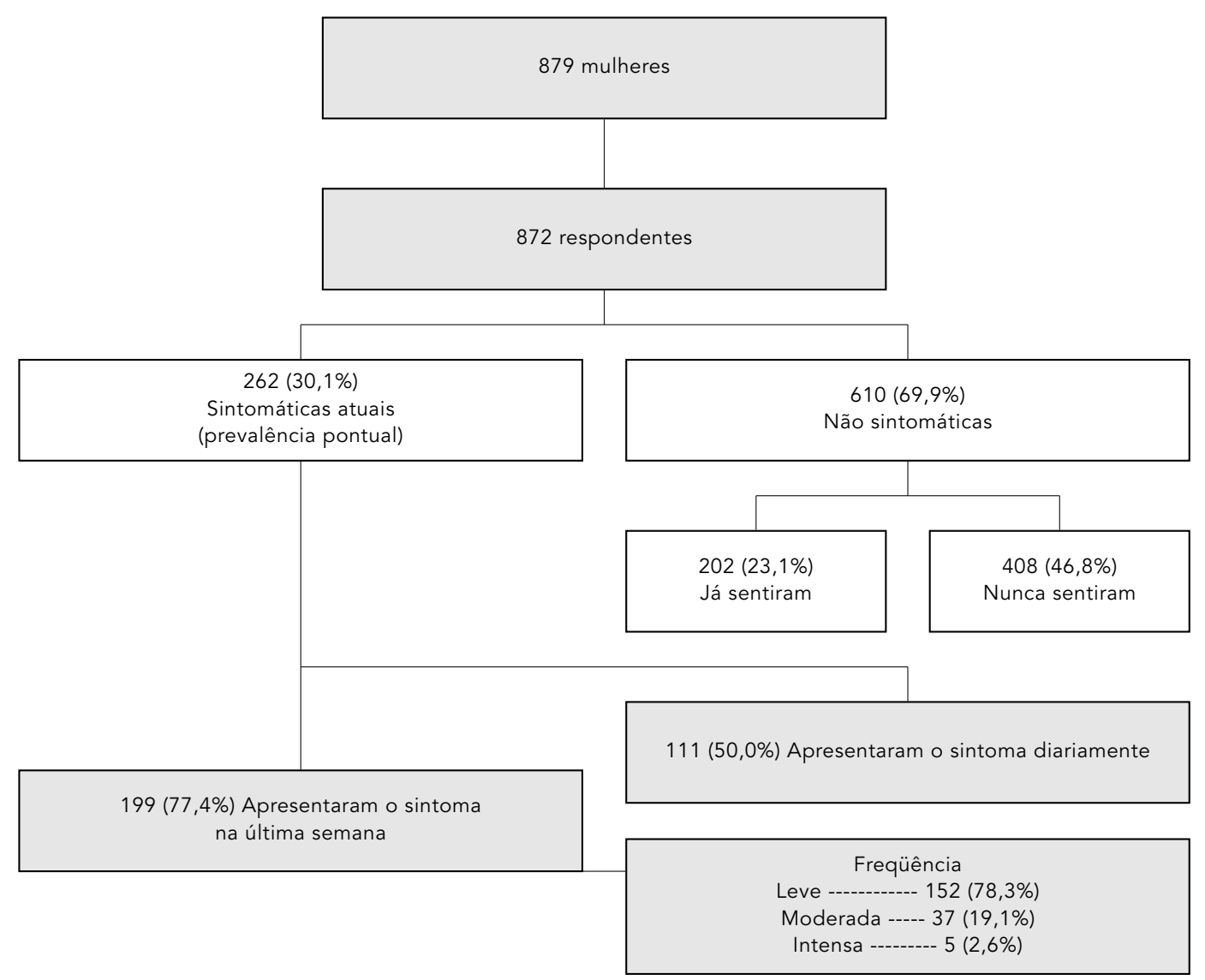

A Tabela 3 apresenta os resultados da análise bivariada e multivariada das variáveis de primeiro e segundo níveis. As mulheres em pósmenopausa apresentaram $\mathrm{RP}=2,66(1,42-4,99)$ para apresentar o sintoma fogacho; e, entre as mulheres em pré-menopausa, o RP foi de 2,33 (1,24-4,38), tendo as mulheres em pós-climatério como categoria de referência. A idade mostrou associação bruta com o desfecho $(p=0,02)$, sendo que as razões de prevalência apresentaram uma distribuição em " $u$ " invertido. O risco de fogachos foi crescente entre as mulheres de 45 até 54 anos. Dos 55 aos 64 anos de idade, as razões de prevalência brutas foram decrescendo de $1,13(0,78-1,63)$, no grupo de 55 a 59 anos, a $0,90(0,58-1,42)$, no grupo de 60 a 64 anos. A faixa etária de 65 a 69 anos foi protetora para o sintoma ( $R P=0,56$; IC95\%: 0,32-0,98). Na análise multivariada, a associação com o desfecho manteve-se significante ( $p=0,03)$, como também o padrão em "u" invertido. As mulheres de maior risco de apresentar fogachos estavam na faixa de 45 a 49 anos e de 50 a 54 anos, com RP ajustados de 1,34 e 1,42, respectivamente. Após os 55 anos, observou-se um declínio do risco, sendo que, entre as mulheres de 65 a 69 anos, o risco de apresentar fogachos foi $60,0 \%$ menor do que entre as mulheres de 40 a 44 anos.

A variável cor da pele apresentou associação bruta com o desfecho ( $p=0,004)$, sendo que as não brancas apresentaram risco $40,0 \%$ maior de apresentar fogachos, quando comparadas com as brancas. $\mathrm{Na}$ análise multivariada, esse efeito diminuiu ( $R P=1,24)$, e a significância estatística ficou limítrofe $(p=0,05)$. A situação conjugal, na análise bruta, não se mostrou associada $(p=0,06)$ com fogachos. Após o ajuste para as outras variáveis do modelo, no en- 
Razões de prevalência brutas e ajustadas para presença de fogachos atuais, de acordo com o status menopausal e com características sócio-econômicas, demográficas, biológicas, comportamentais e de manejo médico da mulher. Pelotas, Rio Grande do Sul, Brasil, 2002.

\begin{tabular}{|c|c|c|c|c|}
\hline Variável & RP bruta & IC $95 \%$ & RP ajustada & IC95\% \\
\hline \multicolumn{5}{|l|}{ Primeiro nível } \\
\hline \multicolumn{5}{|c|}{ Status menopausal $(n=872)^{*}$} \\
\hline Pós-climatério & 1,00 & & 1,00 & \\
\hline Pós-menopausa & 2,66 & $1,42-4,99$ & 2,66 & $1,42-4,99$ \\
\hline Pré-menopausa & 2,33 & $1,24-4,38$ & 2,33 & $1,24-4,38$ \\
\hline \multicolumn{5}{|l|}{ Segundo nível** } \\
\hline \multicolumn{5}{|c|}{ Idade (anos completos) $(n=879)$} \\
\hline $40-44$ & 1,00 & & 1,00 & \\
\hline $45-49$ & 1,34 & $0,98-1,84$ & 1,34 & $0,99-1,83$ \\
\hline $50-54$ & 1,60 & $1,19-2,15$ & 1,42 & $1,00-2,01$ \\
\hline $55-59$ & 1,13 & $0,78-1,63$ & 0,94 & $0,61-1,46$ \\
\hline $60-64$ & 0,90 & $0,58-1,42$ & 0,68 & $0,40-1,16$ \\
\hline $65-69$ & 0,56 & $0,32-0,98$ & 0,39 & $0,18-0,87$ \\
\hline \multicolumn{5}{|l|}{ Cor da pele $(n=879)$} \\
\hline Branca & 1,00 & & 1,00 & \\
\hline Não branca & 1,40 & $1,11-1,77$ & 1,24 & $0,98-1,57$ \\
\hline \multicolumn{5}{|c|}{ Situação conjugal ( $n=879)$} \\
\hline Com companheiro & 1,00 & & 1,00 & \\
\hline Sem companheiro & 0,81 & $0,65-1,00$ & 0,80 & $0,64-0,99$ \\
\hline \multicolumn{5}{|c|}{ Escolaridade (anos) $(n=877)^{\star}$} \\
\hline $12-18$ & 1,00 & & 1,00 & \\
\hline $9-11$ & 1,48 & $0,94-2,31$ & 1,12 & $0,69-1,83$ \\
\hline $5-8$ & 1,66 & $1,12-2,47$ & 1,10 & $0,68-1,78$ \\
\hline $1-4$ & 1,82 & $1,22-2,71$ & 1,17 & $0,70-1,94$ \\
\hline 0 & 1,22 & $0,72-2,06$ & 0,82 & $0,43-1,56$ \\
\hline \multicolumn{5}{|c|}{ Trabalho remunerado $(n=879)$} \\
\hline Sim & 1,00 & & 1,00 & \\
\hline Não & 1,03 & $0,84-1,27$ & 1,02 & $0,83-1,26$ \\
\hline \multicolumn{5}{|c|}{ Renda da mulher (salários mínimos) $(n=387)^{\star \star \star}$} \\
\hline$\geq 5,1$ & 1,00 & & 1,00 & \\
\hline $3,1-5$ & 1,25 & $0,62-2,50$ & 0,93 & $0,46-1,89$ \\
\hline $1,1-3$ & 1,18 & $0,66-2,10$ & 0,89 & $0,43-1,83$ \\
\hline $0,6-1$ & 1,75 & $1,00-3,07$ & 1,25 & $0,59-2,68$ \\
\hline$\leq 0,5$ & 1,59 & $0,83-3,05$ & 1,18 & $0,50-2,79$ \\
\hline \multicolumn{5}{|l|}{$\operatorname{ANEP}(n=877)^{\star}$} \\
\hline A & 1,00 & & 1,00 & \\
\hline B & 1,12 & $0,55-2,25$ & 1,17 & $0,59-2,32$ \\
\hline C & 1,73 & $0,91-3,32$ & 1,90 & $1,01-3,56$ \\
\hline$D$ & 1,90 & $0,99-3,63$ & 2,15 & $1,14-4,03$ \\
\hline$E$ & 1,92 & $0,90-4,10$ & 2,16 & $1,02-4,54$ \\
\hline
\end{tabular}

(continua) 
Tabela 3 (continuação)

\begin{tabular}{|c|c|c|c|c|}
\hline Variável & RP bruta & IC95\% & RP ajustada & IC95\% \\
\hline \multicolumn{5}{|l|}{ Terceiro nível\# } \\
\hline \multicolumn{5}{|c|}{ Início da atividade sexual (anos) $(n=821)^{*}$} \\
\hline$\leq 15$ & 1,00 & & 1,00 & \\
\hline $16-20$ & 0,85 & $0,61-1,18$ & 0,93 & $0,66-1,31$ \\
\hline $21-25$ & 0,75 & $0,51-1,09$ & 0,68 & $0,57-1,28$ \\
\hline$\geq 26$ & 0,78 & $0,50-1,23$ & 0,98 & $0,62-1,55$ \\
\hline \multicolumn{5}{|c|}{ Uso de anticoncepção hormonal (após 40 anos) $(n=875)^{*}$} \\
\hline Sim & 1,00 & & 1,00 & \\
\hline Não & 1,26 & $0,98-1,62$ & 1,40 & $1,07-1,82$ \\
\hline \multicolumn{5}{|c|}{ Hábito de fumar $(n=879)$} \\
\hline Não & 1,00 & & 1,00 & \\
\hline Sim & 1,39 & $1,11-1,75$ & 1,32 & $1,03-1,68$ \\
\hline Ex-fumante & 1,21 & $0,93-1,59$ & 1,18 & $0,89-1,55$ \\
\hline \multicolumn{5}{|c|}{ Índice de massa corporal (referido atual) $(n=823)^{\star}$} \\
\hline Normal $(<25)$ & 1,00 & & 1,00 & \\
\hline Sobrepeso (25-29) & 1,32 & $1,03-1,69$ & 1,32 & $1,03-1,68$ \\
\hline Obeso $(>30)$ & 1,39 & $1,06-1,83$ & 1,39 & $1,06-1,83$ \\
\hline \multicolumn{5}{|l|}{ Quarto nível\#\# } \\
\hline \multicolumn{5}{|c|}{ Idade da menarca (anos) $(\mathrm{n}=862)^{*}$} \\
\hline $16-18$ & 1,00 & & 1,00 & \\
\hline $13-15$ & 1,43 & $0,83-2,46$ & 1,67 & $0,91-3,05$ \\
\hline $8-12$ & 1,49 & $0,86-2,57$ & 1,64 & $0,90-3,01$ \\
\hline
\end{tabular}

RP = razão de prevalência; IC95\% = intervalo de confiança 95\%; ANEP = Associação Nacional de Empresas de Pesquisa.

* Variável com perda de informação.

** Ajustado para variáveis do mesmo nível e para status menopausal.

$\star \star \star$ Somente mulheres com renda própria.

\# Ajustado para variáveis do mesmo nível e variáveis do primeiro e segundo níveis.

\#\# Ajustado para as variáveis do primeiro, segundo e terceiro níveis.

tanto, detectou-se confusão negativa sobre o efeito da situação conjugal, e as mulheres sem companheiro apresentaram risco $20,0 \%$ menor de apresentar o sintoma, quando comparadas com as que viviam com companheiro.

Quanto à escolaridade da mulher, observouse um efeito linear inverso com a presença de sintomas. As mulheres com 9 a 11 anos de escolaridade apresentaram uma probabilidade $48,0 \%$ maior de relatarem fogachos, quando comparadas a mulheres com 12 anos de educação formal. Entre as mulheres com 5 a 8 anos e 1 a 4 anos de escolaridade, o risco aumentou em $66,0 \%$ e $82,0 \%$, respectivamente, em relação à categoria de base (mulheres com 12-18 anos de educação formal). Após análise multivariada, com ajuste para status menopausal, idade, cor da pele, situação conjugal, trabalho remunerado, renda da mulher e status sócio-econômico, a escolaridade não manteve associação estatisticamente significativa com o desfecho $(p=0,61)$, sendo removida do modelo de análise.
O trabalho remunerado da mulher não mostrou associação estatisticamente significante com a presença de fogachos $(p=0,73)$. Após ajuste para as variáveis de primeiro e segundo níveis, esse achado não se modificou $(\mathrm{p}=0,85)$. $\mathrm{Na}$ análise bruta, a renda da mulher mostrou associação estatisticamente significativa com a presença do sintoma ( $p=0,03$ ), mostrando que as mulheres com renda menor ou igual a cinco salários mínimos tinham maior risco de apresentar fogachos, quando comparadas com as mulheres que ganhavam mais de cinco salários mínimos. Após ajuste para status menopausal, idade, cor da pele, situação conjugal, escolaridade, trabalho remunerado e status sócio-econômico, no entanto, a renda da mulher perdeu a significância estatística $(\mathrm{p}=0,53)$. O status sócio-econômico, na análise bivariada, mostrou-se estatisticamente significante $(p=0,001)$, havendo tendência linear de aumento do risco de fogachos, quanto menos favorável a status sócio-econômico. Após análise multivariada, 
mulheres do status sócio-econômico E apresentaram um risco mais do que duas vezes maior de apresentar fogachos $(\mathrm{RP}=2,16)$ do que as mulheres da classe $\mathrm{A}$, tomadas como referência.

A idade de início da atividade sexual não apresentou associação bruta com o desfecho $(\mathrm{p}=0,48)$. Após ajuste para as variáveis de mesmo nível e para o status menopausal, idade, cor da pele, situação conjugal e status sócio-econômico, esse resultado manteve-se inalterado $(p=0,75)$. Na análise bivariada, o uso de anticoncepção hormonal, após os quarenta anos, não mostrou associação estatisticamente significante com a presença de fogachos $(p=0,06)$. Após análise multivariada, ajustando-se para as variáveis do mesmo nível e para o status menopausal, idade, cor da pele, situação conjugal e status sócio-econômico, detectou-se confusão negativa sobre o efeito dessa variável. Comparadas às usuárias, as mulheres que não usavam anticoncepção hormonal apresentaram risco $40,0 \%$ maior de terem fogachos $\mathrm{RP}=1,40$ (1,07 a 1,82). O hábito de fumar, na análise bruta, mostrou associação com a presença de sintomas $(p=0,01)$, sendo que as mulheres fumantes tinham um risco $39,0 \%$ maior de terem fogachos. Após ajuste do modelo, a variável perdeu a significância $(p=0,10)$, sendo mantida apenas para controle de confusão.

Quanto ao IMC referido, observou-se tendência linear direta com o risco para a presença de fogachos, tendo esse aumentado diretamente com o sobrepeso e a obesidade $(\mathrm{RP}=1,32$ e 1,39 , respectivamente) $(\mathrm{p}=0,01)$. A análise ajustada não modificou esse resultado, sendo que as mulheres obesas apresentaram risco $40,0 \%$ maior de desenvolverem fogachos quando comparadas às de peso normal. A variável de quarto nível, idade da menarca, não apresentou associação bruta $(p=0,35)$ com o desfecho, mantendo-se também sem associação estatisticamente significativa, após ajuste para o primeiro, segundo e terceiro níveis.

\section{Discussão}

Este estudo mostrou que, em uma amostra da população de mulheres de 40-69 anos, a prevalência ponto de fogachos foi de $30,1 \%$. Apresentaram maior risco de ter o sintoma as mulheres pós-menopáusicas, com 50-54 anos de idade, não brancas, vivendo com companheiro, pertencentes aos estratos mais pobres da população e com IMC compatível com sobrepeso e obesidade. O uso de métodos anticoncepcionais hormonais, após os quarenta anos de idade, apresentou efeito protetor sobre a ocorrência de fogachos.

Este estudo tem algumas limitações. Primeiro, por tratar-se de estudo transversal, não foi possível incluir, no status menopausal, a categoria de peri-menopausa, que compreende o período em que se iniciam os sintomas e/ou a irregularidade menstrual até um ano após a menopausa 2 , fase de maior vulnerabilidade ao sintoma. Tal problema é inevitável nesse tipo de estudo, uma vez que o diagnóstico de menopausa somente pode ser feito a posteriori, após um ano da ausência de menstruação. Em segundo lugar, na categoria de pré-menopausa, foram incluídas mulheres de fato em prémenopausa (mulheres com quarenta anos ou mais de idade e que ainda não apresentavam sintomas ou ciclos menstruais irregulares) e mulheres em peri-menopausa (mulheres com ou sem sintomas e com ausência de menstruação há menos de um ano). O efeito desse erro de classificação sobre a medida de prevalência é imprevisível, uma vez que não era possível saber o número real de mulheres em cada uma dessas fases do climatério. Do ponto de vista do planejamento e gerenciamento de programas de saúde da mulher, no entanto, tais limitações poderão ser superáveis. É satisfatório saber que o serviço de saúde precisa estar preparado para atender um contingente aproximadamente de $30,0 \%$ de mulheres, na faixa etária de 40 a 69 anos, por sintomas relacionados à menopausa.

Uma outra limitação é que o viés de memória pode ter ocorrido, principalmente nas mulheres mais velhas, por se tratarem de dados colhidos com ocorrência no passado.

A história materna de fogachos, a paridade e o tipo de menopausa (natural ou cirúrgica), características potencialmente importantes na ocorrência de fogachos, não puderam ser investigados. Sendo este um estudo em consórcio com outros pesquisadores, havia uma limitação previamente pactuada quanto ao número de perguntas.

Por outro lado, o método de seleção das mulheres de 40 a 69 anos garantiu uma amostra representativa da população, e o baixo percentual de perdas e recusas diminuiu a possibilidade de viés de seleção. O número de mulheres ( $\mathrm{n}=879$ ) garantiu ao estudo um poder de $80,0 \%$ de detectar risco relativo $\geq 2$, não existindo assim problemas de tamanho amostral para as variáveis com associações dessa magnitude.

É difícil estabelecer comparações entre o atual resultado e os achados de outros autores devido à ampla variedade de definições do status menopausal utilizadas, a diferentes faixas 
etárias das mulheres e à procedência da amostra (populacional ou de serviços) 3,4,12,13,23,24,25, $26,27,28$. Embora tenham sido descritas baixas prevalências de fogachos entre mulheres do leste asiático 3,23 , a maioria dos estudos relatam valores acima de $50,0 \% 4,12,22,24,26,27,28$. Os estudos que detectaram altas prevalências foram realizados com amostras de mulheres provenientes de clínicas ginecológicas ou hospitais, mulheres em pós-menopausa e mulheres de faixa etária de maior risco para o sintoma.

No atual estudo, observou-se associação estatisticamente significativa do status menopausal com a presença do sintoma fogacho. As mulheres em pós-menopausa tiveram o maior risco de desenvolver o sintoma, quando comparadas com as em pós-climatério. Em um estudo realizado em Roterdã, Holanda 8, a prevalência de fogachos também variou de acordo com a categoria menopausal, tendo as mulheres em peri-menopausa a maior prevalência $(69,1 \%)$, seguidas das mulheres em pós-menopausa, com $61,3 \%$. Resultados semelhantes foram encontrados em estudos com mulheres brasileiras e chilenas 4,29. Estudos realizados na Austrália 30,31 mostraram maior prevalência de sintomas na pós-menopausa, o que também foi encontrado por den Tokelaar et al. 32 , na Holanda. Foi observada, entre mulheres suecas 33 , em estudo de 1995, maior prevalência de fogachos $(51,0 \%)$ na peri-menopausa e de $40,0 \%$ na pós-menopausa.

Quanto à idade, a maior prevalência entre as mulheres de 50 a 54 anos é consistente ao encontrado por Rodstrom et al. 7, para essa faixa etária $(60,0 \%)$.

$\mathrm{O}$ achado de associação limítrofe entre a cor da pele e a presença atual de fogachos foi consistente com o achado de um estudo com mulheres norte-americanas 13. Um outro estudo mostrou diferença de prevalência de fogachos, de acordo com a cor da pele, sendo esses mais prevalentes entre as negras 11. É possível que o resultado do atual estudo seja decorrente de erro de classificação da variável cor da pele ou do tamanho amostral, com conseqüente falta de poder, uma vez que, no estudo que detectou associação 11 , a razão de odds foi da ordem de 1,56.

A situação conjugal mostrou que mulheres sem companheiro têm proteção de $20,0 \%$ na ocorrência de fogachos. Na revisão da literatura, não foram encontrados estudos que mostrem tal associação. Há a necessidade de que outros estudos, usando diferentes metodologias, explorem mais detalhadamente esse achado.

Observou-se tendência linear de diminuição da prevalência de fogachos quanto maior o status sócio-econômico, mesmo após ajuste para possíveis fatores de confusão, sendo as mulheres da classe $\mathrm{E}$ as mais acometidas pelo sintoma. Estudo em mulheres de baixo nível sócio-econômico, na Tailândia, mostrou alta prevalência de sintomas associados com baixa escolaridade e status sócio-econômico 22.

Apesar de muitos estudos mostrarem associação do hábito de fumar com fogachos 9,10,12, o presente estudo, após análise ajustada, não mostrou associação estatisticamente significativa.

Pelas bases teóricas encontradas em bibliografia específica de ginecologia 34 , as mulheres obesas seriam as menos sintomáticas, pela conversão periférica de androstenediona em estrona no tecido gorduroso, capacidade essa aumentada na pós-menopausa. O atual estudo, no entanto, detectou aumento de risco para fogachos nas mulheres com sobrepeso ou obesas. Outros autores, mais recentemente, descreveram achados semelhantes 9,13. Tal resultado, no entanto, deve ser considerado com cuidado, uma vez que o IMC foi calculado com base no peso e altura referidos pela mulher, na data da entrevista. Tal aspecto metodológico tem duas implicações. Primeiro, no que diz respeito à precisão das medidas, é possível que as entrevistadas tenham errado o peso para mais, o que resultaria em aumento no IMC. Há, no entanto, evidências na literatura de que o peso referido pela população adulta tem, em geral, alta correlação com o peso medido 35,36 , tornando improvável essa fonte de erro. A segunda implicação diz respeito ao viés de causalidade reversa. O peso referido é o atual, não sendo possível, por força do delineamento do estudo, afirmar que as medidas antropométricas da mulher eram as mesmas antes do aparecimento dos fogachos.

O uso de anticoncepção hormonal, após os quarenta anos, associou-se a um menor risco de fogachos, provavelmente por se tratar de medicação hormonal. Lamentavelmente, não foram encontrados na literatura outros estudos que tivessem explorado essa variável. Um outro aspecto observado entre as mulheres entrevistadas foi que, dentre as atualmente sintomáticas, cerca da metade $(43,0 \%)$ realiza algum tipo de tratamento, hormonal ou não. Do total de mulheres, $13,1 \%$ estavam fazendo tratamento para menopausa, valor esse semelhante ao encontrado por estudo de 1994, em Manila (Filipinas), em Beirute e no México, no ano de 1994 (respectivamente, 11,6\%, 15,0\% e 12,5\%). Nesse momento em que as medicações usadas para os sintomas do climatério, especialmente as hormonais, vêm sendo questionadas quanto 
aos riscos e benefícios, torna-se importante buscar formas alternativas de manejo 37 . Este estudo levanta a hipótese de que programas de redução do peso, a exemplo de uma intervenção sueca com promoção de atividade física entre mulheres de 45-55 anos de idade, que resultou em uma redução de 50,0\% na prevalência de fogachos em relação ao grupo controle 2 , poderão ser testados, com vistas ao controle e prevenção de fogachos.

Tais achados têm implicações importantes para os serviços de saúde. A população feminina, com mais de quarenta anos de idade, é um grupo populacional crescente, à medida que aumenta a expectativa de vida. Além disso, um estudo transversal, de base populacional, realizado em Campinas, São Paulo, mostrou que $80,0 \%$ das mulheres de 45 a 60 anos de idade buscavam atendimento médico por irregularidade menstrual e sintomas do climatério 38 . A despeito dessa alta demanda, até o momento, o planejamento familiar e o cuidado pré-natal têm sido as atividades centrais, no setor público, do cuidado à saúde da mulher. É necessário que os serviços de saúde se organizem para manejar, de forma programada, o período pósreprodutivo da vida da mulher. A freqüência e a intensidade dos fogachos, bem como outros sintomas prevalentes no climatério, que comprometem a qualidade de vida da mulher, demandam uma atenção especial por parte dos serviços. Para isso, recomenda-se que outros estudos sejam realizados de forma a investigar plenamente as características e os determinantes da saúde da mulher após os quarenta anos.

\section{Resumo}

Com o objetivo de determinar a prevalência de fogachos e fatores associados, em mulheres entre 40 e 69 anos de idade, provenientes de uma amostra representativa da população da cidade de Pelotas, Rio Grande do Sul, Brasil, realizou-se um estudo transversal. O processo de amostragem foi em múltiplos estágios, e a coleta de dados, realizada através de entrevista e questionário auto-aplicado. Foram incluidas 879 mulheres. A prevalência ponto e a prevalência de episódio de fogachos foram, respectivamente, de $30,1 \%$ e 53,2\%. Os fatores estatisticamente associados a fogachos atuais foram a categoria menopausal de pré-menopausa $(R P=$ 2,33) e pós-menopausa $(R P=2,66)$; idade de 45 a 49 anos $(R P=1,34)$ e de 50 a 54 anos $(R P=1,42)$; status sócio-econômico mais baixo $(R P=2,16)$; não uso de anticoncepção hormonal após os 40 anos $(R P=1,40)$; obesidade $(R P=1,39)$; sobrepeso $(R P=1,32)$ e viver sem companheiro $(R P=0,80)$. Dada a alta prevalência do sintoma, é necessário que os serviços de saúde se organizem para lidar com a mulher climatérica.

Climatério; Fogachos; Menopausa

\section{Colaboradores}

I. K. T. Sclowitz estruturou o conteúdo do texto, analisou os dados e redigiu a versão do artigo. I. S. Santos orientou, revisou e implementou as modificações necessárias. M. F. Silveira co-orientou e revisou o artigo.

\section{Referências}

1. Utian WH. The International Menopause Society menopause-related terminology definitions. Climateric 1999; 2:284-6.

2. World Health Organization. Research on the menopause in the 1990s. Geneva: World Health Organization; 1996. (Technical Report Series 866).

3. Tang GW. The climacteric of Chinese factory workers. Maturitas 1994; 19:177-82.

4. Halbe HW, Fonseca AM, Assis JDS, Vitória SM, Arie MHA, Elias DS, et al. Aspectos epidemiológicos e clínicos em 1.319 pacientes climatéricas. Revista de Ginecologia e Obstetrícia 1989; 1:182-94.

5. Boulet MJ, Oddens BJ, Lehert P, Vemer HM, Vissel A. Climacteric and menopause in seven Southeast Asian countries. Maturitas 1994; 19:157-76.

6. Stadberg E, Mattsson LA, Milson I. The prevalence and severity of climacteric symptoms and the use of different treatment regimens in a Swedish population. Acta Ostet Gynecol Scand 1997; 76:442-8.

7. Rodstrom K, Bengtsson C, Lissner L, Milsom I, Sundh V, Bjorkelund C. A longitudinal study of the treatment of hot flushes: the population study of women in Gothenburg during a quarter of a century. Menopause 2002; 9:156-61.

8. Groeneveld FP, Bareman FP, Barentsen R, Dokter HJ, Drogendijk AC, Hoes AW. Vasomotor symptoms and well being in the climacteric years. Maturitas 1996; 23: 293-9.

9. Gold EB, Sternfeld B, Kelsey JL, Brown C, Mouton C, Reame N, et al. Relation of demographic and lifestyle factors to symptoms in a multi-racial/ ethnic population of women 40-55 years of age. Am J Epidemiol 2000; 152:463-73. 
10. Obermeyer CM, Schulein M, Hajji N, Azelmat M. Menopause in Morocco: symptomatology and medical management. Maturitas 2002; 41:87-95.

11. Grisso JA, Freeman EW, Maurin E, Garcia-Espana B, Berlin JA. Racial differences in menopause information and the experience of hot flashes. J Gen Intern Med 1999; 14:98-103.

12. Staropoli CA, Flaws JA, Bush TL, Moulton AW. Predictors of menopausal hot flushes. J Womens Health 1998; 7:1149-55.

13. Freeman EW, Sammel MD, Grisso JA, Battistini M, Garcia-Espagna B, Hollander L. Hot flashes in the late reproductive years: risk factors for Africa American and Caucasian women. J Womens Health Gend Based Med 2001; 10:67-76.

14. Bertoldi AD, Barros AJ, Hallal PC, Lima RC. Utilização de medicamentos em adultos: prevalência e determinantes individuais. Rev Saúde Pública 2004; 38:228-38.

15. Carret ML, Fassa AG, Silveira DS, Bertoldi AD, Hallal PC. Sintomas de doenças sexualmente transmissíveis em adultos: prevalência e fatores de risco. Rev Saúde Pública 2004; 38:76-84.

16. Domingues MR, Araújo CL, Gigante DP. Conhecimento e percepção sobre exercício físico em uma população adulta urbana do Sul do Brasil. Cad Saúde Pública 2004; 20:204-15.

17. Gazalle FK, Lima MS, Tavares BF, Hallal PC. Sintomas depressivos e fatores associados em população idosa no Sul do Brasil. Rev Saúde Pública 2004; 38:365-71.

18. Hallal PC, Victora CG, Wells JC, Lima RC. Physical inactivity: prevalence and associated variables in Brazilian adults. Med Sci Sports Exerc 2003; 35: 1894-900.

19. Instituto Brasileiro de Geografia e Estatística. Contagem da população - 1996. Rio de Janeiro: Instituto Brasileiro de Geografia e Estatística; 1996.

20. Associação Nacional de Empresas de Pesquisa. Critério de classificação econômica do Brasil. Brasília: Associação Nacional de Empresas de Pesquisa; 2002.

21. Traissac CP, Martin-Prevel Y, Delpeuch F, Maire B. Régression logistique vs autres modèles linéaires generalizes pour l'estimation de rapports de prévalences. Rev Epidemiol Sante Publique 1999; 47:593-604.

22. Chaikittisilpa S, Limpaphayom K, Chompootweep $S$, Taechakraichana N. Symptoms and problems of menopausal women in Klong Toey slum. J Med Assoc Thai 1997; 80:257-61.

23. Ho SC, Chan SG, Yip YB, Cheng A, Yi Q, Chan C. Menopausal symptoms and symptom clustering in Chinese women. Maturitas 1999; 33:219-27.

24. Nedstrand E, Pertl J, Hammar M. Climacteric symptoms in a postmenopausal Czech population. Maturitas 1996; 23:85-9.
25. Stenberg A, Heimer G, Ulmsten U, Cnattingius S. Prevalence of genitourinary and other climacteric symptoms in 61-year-old women. Maturitas 1996; 24:31-6.

26. Schwingl PJ, Hulka BS, Harlow SD. Risk factors for menopausal hot flashes. Obstet Gynecol 1994; 84:29-34.

27. Velasco-Murillo V, Navarrete-Hernandez E, Ojeda-Mijares RI, Pozos-Cavanzo JL, Camacho-Rodriquez MA, Cardona-Perez JA. Experience and knowledge about climacteric and menopause in woman in Mexico City. Gac Med Mex 2000; 136: $555-64$.

28. von Muhlen DG, Kritz-Silverstein D, Barrett-Connor E. A community-based study of menopause symptoms and estrogen replacement in older women. Maturitas 1995; 22:71-8.

29. Blumel MJMEM, Gramegna-Sougarret GM, Tacla FX, Sepúlveda MH, Brandt AA. Prevalencia de sintomas psíquicos y vasomotores en diferentes periodos del climaterio. Rev Chil Obstet Ginecol 1997; 62:412-8.

30. Dennerstein L, Dudley EC, Hopper JL, Guthrie JR, Burger HG. A prospective population-based study of menopausal symptoms. Obstet Gynecol 2000; 96:351-8.

31. Guthrie JDL, Hopper JL, Burger HG. Hot flushes, menstrual status, and hormone levels in a population-based sample of midlife women. Obstet Gynecol 1996; 88:437-42.

32. den Tonkelaar I, Seidell JC, van Noord PA. Obesity and fat distribution in relation to hot flashes in Dutch women from the DOM-project. Maturitas 1996; 23:301-5.

33. Bardel A, Wallander MA, Svardsudd K. Hormone replacement therapy and symptom reporting in menopausal women: a population-based study of 35-65-year-old women in mid-Sweden. Maturitas 2002; 41:7-15.

34. Halbe HW. Tratado de ginecologia. In: Halbe HW, Fonseca AM, organizadores. Síndrome do climatério. São Paulo: Editora Roca; 1998. p. 1243-58.

35. Stewart AJR, Ford M, Beagleohole R. Underestimation of relative weight by use of self-reported height and weight. Am J Epidemiol 1987; 125:122-6.

36. Schmidt MDB, Tavares M, Polanczyk C, Pellanda L, Zimmer P. Validity of self-reported weight - a study of urban Brazilian adults. Rev Saúde Pública $1993 ; 27: 271-6$.

37. Women's Health Initiative. Risks and benefits of estrogen plus progestin in healthy postmenopausal women. JAMA 2002; 288:321-68.

38. Pedro AO, Pinto Neto AM, Costa-Paiva L, Osis MJ, Hardy E. Climacteric women seeking medical care. Rev Saúde Pública 2002; 36:484-90.

Recebido em 12/Mar/2004

Versão final reapresentada em 27/Ago/2004

Aprovado em 01/Set/2004 